R. Heiden hain: Unters, über d. Einfluss đ. nv. vag. a. d. Herzthätigkeit. 383

(Aus dem physiologischen Institut zu Breslau.)

\title{
Untersuchungen über den Einfluss des nv. vagus auf die Herzthätigkeit.
}

Von

\section{R. Heidenhain.}

(Hierzu Tafel IX und X.)

\section{§ 1. Methode und nächster Zweck der Untersuchnng.}

Vagus und Herz, - seit den Tagen Ed. Weber's bis auf die unsrigen ein Thema von unerschöpflicher Anziehungskraft für die Untersuchung! War doch in der Einwirkung jenes Nerven auf das Herz das erste Beispiel einer Hemmungswirkung gegeben, dem erst nach längerer Zeit andre folgten. Die Hoffnung, an jenem scheinbar einfachsten Falle dem Wesen dieser friberhin unbekannten Nervenwirkungen näher zu treten, hat wieder und wieder zur experimentellen Bearbeitung desselben geführt, - und doch stehen wir heute vor dem Räthsel, dass ein Nerv eine motorische Thätigkeit unterdrücken könne, in ziemlich derselben Rathlosigkeit, wie damals, als es zuerst aufgedeckt ward.

Seit meinen in Gemeinschaft mit $\mathrm{B} u \mathrm{~b}$ n off angestellten Untersuchungen über die motorisehen Eigenschaften der grauen Hirnrinde mit den Erscheinungen der Erregung und Hemmung unausgesetzt beschäftigt ${ }^{1}$ ), hoffte ich an dem Vagus gewisse bis dahin immer noch zweifelhafte Fragen auf diesem Gebiete einer Lösung

1) N. Bubn off und R. Heiden hain, über Erregungs- und Hemmungsvorgänge innerhalb der motorischen Hirncentren. Pflügers Archiv XXVI. 1881. S. 193.

E. Pflūger, Arehiv f. Physlologie. Bd. XXvII. 
näher führen zu können. Es galt zu entscheiden, einerseits ob der Vagus auf das Herz ausser der unbestrittenen Hemmungswirkung eine bis in unsere Tage oft bestrittene Erregungswirkung austibe; demnächst, wenn die letztere sich mit Sicherheit feststellen liess, ob diese beiderlei Wirkungen von denselben oder von verschiednen anatomischen Elementen abbingen ${ }^{1}$ ). Die Untersuchung hat in ihrem Verlaufe nach manchen Richtungen hin eine ursprünglich nicht beabsichtigte Ausdehnung gewonnen; eine kurze Darstellung ihrer wesentlichen Resultate ist der Zweck des vorliegenden Aufsatzes. -

Als Versuchsobject diente der Frosch. Das Herz wurde im Zusammenhange mit den beiden nv. vagis ausgeschnitten und horizontal auf eine Glasplatte gelagert, um die Ventrikelpulse mittelst eines äusserst leichten Fühlhebels ${ }^{2}$ ) auf die Trommel des Baltzar'schen Kymographions zu zeichnen. Der von mir benutzte Hebel ist dem Ranvier'schen (s. unten) sehr ähnlich, aus einem Strohhalme gefertigt, nahezu äquilibrirt, und stützt sich auf den Ventrikel mittelst eines Knochenplättchens, welches sich auf der Oberfläche des Herzens vermöge seiner Hygroseopicität leicht ansaugt, so dass es auf derselben hinreichend haftet. Eine ausführlichere Beschreibung des Instrumentchens ist bei seiner Einfachheit wohl unnöthig. Es sei nur bemerkt, dass ich die Hebelaxe in verticaler Richtung verstellbar gemacht habe, um dem Hebel bei der Benutzung von Herzen verschiedenen Umfanges immer nahezu horizontale Anfangsstellung geben zu können, und dass ich besondern Werth auf möglichst feine Einstellung der an dem Hebelende befindlichen Borste zur Pulszeichnung gelegt habe, um die Reibung auf der berussten Papierfläche möglichst zu verringern. Behufs ihrer genauen Anlagerung an die berusste Papierfläche ist die Glasplatte, auf welcher sich der Hebel befindet, (vgl. die Figur bei Ranvier) vermittelst zweier durch Micrometerschraube stellbarer

1) R. Heidenhain, über Erregung und Hemmung. Pflügers Archiv XXVI. 1881 . S. 556 u. 557.

2) Die Contractionen des Froschventrikels sind schon häufig mit Hülfe von Fühlhebeln graphisch dargestellt worden. Vgl. Lauder Brunton, The journal of anatomy and physiology X. 602. 1876. - Marey, travaux de laboratoire I. Année. 1875. S. 44. 1876. - Foster, some effects of upas antiar on the frogs heart. Journ. of anatomy and physiology X. 586. Ranvier, leçons d'anatomie generale 1877-1878. S. 42. Paris 1880. 
Untersuchungen über den Einfluss des nv. vagus auf die Herzthätigkeit. 385

Schlitten in tangentialer und radialer Richtung gegen die Schreibtrommel verschiebbar gemacht. Der Ventrikel muss genau horizontal gelagert sein, was durch untergeschobene Glasplättchen leicht bewerkstelligt werden kann. Alle contractilen Nachbartheile des Herzens, namentlich die Speiseröhre, müssen entfernt werden, um jeder Verschiebung des Herzens bei der Reizung des Vagus vorzubengen. Unterhalb der Pulscurven giebt ein kleiner Electromagnet.Secunden an, ein zweiter zeichnet die Reizungen auf, die Schreibspitzen beider befinden sich vertical unter einander und unter der Schreibspitze des Hebels.

Die Reizung des Vagus geschah theils anf electrischem, theils auf chemischem Wege: electrisch durch einzelne Inductionsschläge (Doppelschläge, durch Oeffnung und sofortige Wiederschliessung des primären Kreises erzengt) oder durch eine tetanisirende Folge derselben, chemisch durch einen Tropfen concentrirter Kochsalzlösung oder durch einen Kochsalzkrystall.

\section{§ 2. Hemmungswirkung des nv. vagus.}

Früher ist bekanntlich die Einwirkung des nv. vagus anf das Herz einseitig dahin definirt worden, dass derselbe die Frequenz der Herzschläge herabsetze. Allein bereits Coats ${ }^{1}$ ) hat in einer aus dem Institute von Ludwig hervorgegangenen Arbeit mitgetheilt, dass unter Umständen die tetanische Reizung jenes Nerven gleichzeitig mit der Pulsfrequenz auch die Energie des einzelnen Herzschlages herabmindern könne. Ja es kam bei seinen Versuchen in freilich nur seltenen Fällen vor, dass die Energie der Ventrikelzusammenziehung in merklichem, wenn auch nur geringem Grade $^{2}$ ) ohne alle Frequenzänderung sank. Die Wirkung auf die Kraft des Ventrikels trat nicht immer in die Erscheinung. Ueber die Ursache des Schwankens seiner Resultate kam Coats nicht in's Klare.

Wenn es mir auch nicht gelungen ist, alle jene Erscheinungen beeinfussenden Bedingungen vollständig zu beherrschen, so legen die folgenden Beobachtungen doch einige der Umstände klar, von welchen es abhängt, ob die Vagusreizung nur die Frequenz oder nur die Energie der Herzschläge herabsetzt oder beide Momente

1) Coats, Berichte der K. Sächs. Gesellsch. der Wissensch. zu Leipzig. Sitzung vom 12. Debr. 1869.

2) Vgl. Curve 5 bei Coats. 
gleichzeitig ändert. Es kommt dabei sowohl auf die Art der Reizung, als auf den Zustand des Herzens an, an welchem die Versuche geschehen.

a) Reizung durch einzelne Inductionsschläge. Sendet man durch den nv. vagus eines noch ganz frischen, auf dem Maximo der Leistungsfähigkeit befindlichen Herzens in grösseren Pausen einzelne Inductionsschläge, während man mit der secundären Rolle des Inductoriums allmählich an die primäre, von den Eisendrahtbündeln befreite Rolle herangeht, so beobachtet man als erste Wirkung eine deutliche, auf wenige Pulse beschränkte Herabsetzung der Pulsgrösse, ohne dass sich vorläufig eine Verlängerung der Ventrikeldiastole merklich machte. Werden die Ströme bei weiter angenäherter secundärer Rolle stärker, so tritt zu der Verkleinerung der systolischen Erhebung der Curve, welche immer auffallender wird, eine Vergrösserung der Pulsintervalle. Beide Veränderungen schreiten mit wachsender Stromesintensität immer mehr fort und werden bei gleicher Stärke der Inductionsschläge um so erheblicher, je länger an dem Herzen experimentirt worden und je mehr damit seine Energie gesunken ist.

Beispiele. Curve I (3. 2. 82). Die kleinen Striche auf der untersten Linie bezeichnen den Augenblick eines Oeffnungs- und Schliessungsschlages, die darïber stehenden Zahlen die Entfernung der secundären Rolle von der primären in Centimetern, bei welcher der Inductionsschlag ausgelöst wurde. Bei dem Schlittenstande 7 und 6 hat die Reizung eine deutliche Verkleinerung der systolischen Erhebung zur Folge, welche erst allmählich wieder zu dem ursprünglichen Werthe anwächst. Bei dem Sehlittenstande 5 fallen die näehsten beiden Pulse $a b$, die folgenden steigen allmählich wieder an. Gleichzeitig sind die zweite und dritte auf die Inductionsschläge folgende Diastole ein wenig verlängert. Bei den Schlittenständen 4 und 3 werden beiderlei Wirkungen stärker ausgeprägt. -

Bei der Fortsetzung dieses Versuches wurden die drei Curven II $a, b, c$ gewonnen, indem bei drei auf einander folgenden Cylinderumdrehungen, an gleicher Stelle der drei iuber einander liegenden Curven Inductionsschläge bei den Schlittenständen 4 und 3 durch den Nerven gesandt wurden. Die Curve a entspricht den ersten, c den letzten Reizungen. Die Energie des Herzens nimmt allmählich ab, denn die absolute Grösse der systolischen Erhebung ist in jeder 
folgenden Curve geringer als in der früheren. Ein Vergleich der drei Reizungen bei dem Sehlittenstande 4 resp. 3 zeigt, dass die Wirkung der Reizung sowohl bezitglich der Verkleinerung der systolischen Erbebung, als der Verlängerung der Diastole mit steigender Ermüdung des Ventrikels immer auffallender wird. Merkwuirdiger Weise ist die Zahl der Pulse, welche erfordert wird, um die Systolegrösse von ihrem kleinsten Werthe aus wieder auf ihren grössten Werth zu heben, fuir alle Reizungen gleich.

b) Reizung durch einige, unmittelbar auf einander folgende Inductionsschläge. Wendet man statt einzelner Doppelströme deren mehrere, unmittelbar auf einander folgende an, so erweist sich ihre Wirkung theils von ihrer Stärke, theils von ihrer Zahl abhängig. Beides springt in die Augen, wenn man die über einander stehenden Curven III ( $a$ und b) mit einander vergleicht. Wenn man festhält, dass von den beiden iiber jedem Reizsignale stehenden Zahlen die erste den Schlittenstand, die zweite die Zahl der Doppelströme bezeichnet (also z. B. 6. 4 bedeutet 4 Doppelströme bei dem Schlittenstande 6), so ergiebt sich aus den beiden Curven ohne weitere Erläuterung der Satz: bei gleicher Stärke der Inductionsströme wächst ihre Einwirkung auf die Systolegrösse und die Diastoledauer mit ihrer Zahl; bei gleicher Zahl der Induetionsströme wächst ihre Einwirkung mit ihrer Stärke. -

Bemerkenswerth ist in den Curven II und III noch die Art des Sinkens und Steigens der Pulsgrösse. So lange die Reizung schwach ist, sinkt dieselbe nicht sofort auf das jedesmalige Minimum, sondern erreicht dasselbe erst durch allmähliche Verkleinerung bis zu etwa dem dritten Schlage nach der Reizung. Ist die letztere dagegen stark, so fällt die Pulsgrösse sofort auf Null, d. h. die nächste Pulse fallen ganz aus (II, b, 3; e 4 und 3 . III 4.6). Das Wiederansteigen geschieht allmählicher, als der Abfall, in Form einer aufsteigenden Treppe. Doch kommt es bei kräftigen Herzen und starker Reizung vor, dass schon der erste auf die Herzpause folgende Schlag mit der ursprünglichen, vor der Reizung bestandenen Grösse wieder einsetzt, wovon Curve IV ein Beispiel giebt.

c) Reizung dureh eine Reihe von Inductionsschlägen, welche in grösseren Pausen aufeinander folgen. Ganz besonders interessant gestaltet sich der Erfolg der Reizung durch 
Doppelschläge, welche in längeren Pausen aufeinander folgen. Bei gliteklicher Wahl der Reizintervalle und der Stromesintensität gelingt es, wenn man die Reizpausen allmählich verkuirzt, die Sy stolegrösse obne Frequenzänderung allmählich sinken zu lassen, bis schliesslich der Herzschlag aufhört, ohne dass vor dem Verschwinden desselben die Pulsintervalle sich verlängert hätten.

Curve V (22. Nov. 81) gibt ein Beispiel dieses merkwürdigen Verhaltens. Wie die Reizsignale auf der untersten Linie zeigen, begann die Reizung (bei dem Sehlittenstande 65) mit einem Intervall von 5 Sec., das bei $b$ auf 4 Sec., bei c anf 3 Sec. und bei $d$ auf 2 Sec. verkürt wurde. Schon der Augenschein lebrt, dass trotz des allmählichen Sinkens der Pulsgrösse auf ein Minimum die Frequenz keine Aenderung erleidet. Eine Auszählung der Pulse ergiebt für je 10 Secunden

vor der Reizung: $5-5$,

während derselben

bei 5 Sec. Intervall: $5-5-5-5$

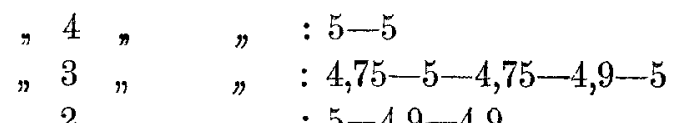

"2,,$: 5-4,9-4,9$

Nach der Reizung: $5-4,75-5-5,25-5-5-5,25-5,25-5,25-5$ $-5,5-5,5$ Pulse.

Eine aufmerksame Betrachtung der Curve lässt aber noch eine weitere wichtige Thatsache erkennen, die übrigens auch schon in den Curven II und III angedeutet ist. Während der Reizung: verkleinert sich nicht bloss die Höhe der Systole, sondern es tritt auch eine stärkere Erschlaffung des Ventrikels ein, als vor und nach der Reizung. Denn wenn man die diastolischen Senkungen, welche die Curve um die Zeit der frequentesten Reizungen macht, durch eine Gerade verbindet und diese nach beiden Seiten hin verlängert, sieht man, dass diese Linie von den diastolischen Senkungen vor und nach der Reizung nicht erreicht wird. Durch die Vagusreizung wird also die diastolische Erschlaffung der Ventrikelmusculatur verstärkt.

Diese Bemerkung ist übrigens nicht neu. Bereits Coats macht in seiner oben erwähnten Arbeit mehrfach darauf aufmerksam, dass die "Ruhespannung" des Herzens während der Vagusreizung 
Untersuchungen ïber den Einfluss des nv. vagus auf die Herzthätigkeit. 389

sinkt. Aubert ${ }^{1}$ ) wirft mit Rücksicht auf die Wahrnehmung, dass die diastolische Ausdehnung eines freigelegten Herzens während der Vagusreizung, bei Erstickung; nach Coffein-Vergiftung u. s. f. ersichtlich erheblicher ausfällt, als unter gewöhnlichen Umständen, die Frage auf, ob die wechselnde Grösse des diastolischen Herzens nicht von einer wechselnden Nachgiebigkeit der Herzwandungen abhängig sei, - eine Frage, die ihn freilich zu der kaum haltbaren Annahme eines besondern kardiotonischen Nervensystems fiuhrt. Während der Redaction des vorliegenden Aufsatzes endlich ging mir eine auszugsweise Mittheilung einer Untersuchung von Gaskell ${ }^{2}$ ) zu, in welcher dieser Autor die Angabe macht, dass Reizung des Vagus den "Tonus" der Ventrikelmusculatur herabsetze. Meine Curven zeigen in Uebereinstimmung mit den Coatsschen, dass die Vagusreizung Vorgänge hervorruft, welche nicht bloss die systolische Zusammenziehung der Ventrikelmusculatur beeinträchtigen, sondern auch die diastolische Erschlaffung begünstigen.

Der Grad der Verkleinerung der Systole ist sowohl von dem zeitlichen Abstande der Inductionsschläge, als von ihrer Stärke abhängig. Je höher die letztere, desto seltenere Reize genügen, um die Pulsgrösse auf ein Minimum herabzudrücken. Unmittelbar vor der Curve V, welche bei dem Schlittenstande 6,5 gewonnen ist, wurde eine andere bei dem Schlittenstande 7,5 gezeichnet. Es bedurfte hier einer Herabsetzung der Reizintervalle auf 0,5 Sec., um zu einer so hochgradigen Verkleinerung der Systolegrösse zu gelangen, wie sie bei dem Schlittenstande 6,5 schon bei einem vierfach so grossen Reizintervall (2 Sec.) erreicht wurde.

Nicht immer gelingt es, dasjenige Verhältniss von Stromstärke und Reizintervall zu treffen, bei welchem die Pulsgrösse allein (ohne die Frequenz) abnimmt: am günstigsten hierfür sind schon etwas ermüdete Herzen. Häufig ändern sich jene beiden Werthe

1) H. Aubert, über den Coffeingehalt des Kaffees und über die Wirkungen des Coffeins. Pflügers Archiv V. 621.

2) Gaskell, on the rhytm of the heart on the frog and on the nature of the action of the vagus nerve. Abstract. Proceedings of the royal society 1881. No. 217. Ich bemerke, dass ich diesen Aufsatz, dessen Ergebnisse sich in manchen Puncten mit den meinigen decken, nach Vollendung meiner Untersuchungen während der Redaction derselben erhalten habe. 
gleichzeitig, und zwar in der Regel in dem Sinne, dass schwächere Ströme von geringerem zeitlichem Abstande in höherem Maasse die Systolegrösse, als die Pulszahl, und stärkere Ströme von grösserem zeitlichem Abstande mehr die Zahl der Pulse, als ihre Grösse beeinflussen.

Die Curven VI ( $a$ und b) zeigen das Verhältniss in klarster Weise: in der Curve a sinkt bei dem Schlittenstande 50, während das Reizintervall von 5 Sec. auf etwa 0,25 Sec. verringert wird, die Pulsgrösse sehr erheblich, die Frequenz sehr wenig; in der unmittelhar darauf bei dem Schlittenstande 45 gewonnene Curve b sinkt schon bei 2 Secunden Intervall die Frequenz enorm, die Grösse der Pulse viel weniger, als vorher. In beiden Curven ist die diastolische Erschlaffung während der Reizung erheblich gesteigert.

\section{$\S$ 3. Vorläufige Discussion der bisherigen Ergebnisse.}

Die mitgetheilten Thatsachen berichtigen gewisse Vorstellungen von der Hemmungswirkung des nv. vagus, welche trotz mancher bereits fritherhin bekannt gewordener widersprechender Erfahrungen noch vielfach in Geltung sind.

Es hat sich gezeigt, dass die Reizung jener Nerven unter gewissen Bedingungen die Systolegrösse des Ventrikels bis zum Verschwinden des Herzschlages verkleinert, ohne dass gleichzeitig die Pulsfrequenz heruntergeht, dass unter anderen Umständen lange diastolische Pausen ohne vorgängige und günstigen Falls sogar ohne nachträgliche Verringerung der Contractionshöhe eintreten können, and dass unter noch anderen Bedingungen ein deprimirender Einfluss sowohl auf die Grösse der Einzelpulse, als anf die Frequenz des Herzschlages zur Beobachtung gelangt.

Es hat sich ferner gezeigt, dass unter allen Umständen jede intensive Vaguserregung, sofern sie iiberhaupt hemmend wirkt, die diastolisehe Erschlaffung des Herzens verstärkt.

Vorgreifend sei hier bemerkt, dass der Einfluss auf die Pulsgrösse nicht bloss bei electrischer Reizung des vagus sich geltend macht, sondern auch bei chemischer Reizung (Kochsalz), sei es des Nervenstammes, sei es des verlängerten Markes, oft genug in die Erscheinung tritt.

Diese Beobachtungen wollen sich mit derjenigen Theorie der 
Untersuchungen über den Einfluss dos nv. vagus auf die Herzthätigkeit. 391

Hemmung, welche von Rosenthal bezïglich der Einwirkung des nv. vagus auf die Athmung entwickelt und von Andern auf die Herzhemmung ibertragen worden ist, schlechterdings nicht reimen. Der Vagus soll nach jener Vorstellung auf die motorischen Herzganglien der Art einwirken, dass er hypothetische Widerstände vergrössert, welche die in jenen Ganglien entstehenden Reize bei ihrem Uebergange auf die motorischen Nervenfasern finden. Daraus ergebe sich einerseits eine Verlängerung der Pulsintervalle, weil längere Zeit erforderlich sei, um die intraganglionären Reize so weit ansehwellen zu lassen, dass sie die vergrösserten Widerstände zu überwinden vermögen. Andererseits ergebe sich eine Verstärkung der Einzelpulse, da die jedesmalige motorische Erregung, weil durch eine vergrösserte Reizsumme bedingt, auch eine verstärkte Einwirkung auf die motorischen Nervenfasern haben müsse. Es hat sich nun aber bei den obigen Beobachtungen in noch viel weiterem Umfange, als bei den Versuchen von Coats, gezeigt, dass bei passender Erregungsweise der nv. vagus die Systolegrösse des Ventrikels bei ungeänderter Frequenz bis zum Erlöschen des Herzschlages verkleinert, - eine mit jener „Widerstandstheorie" nicht zu vereinigende Thatsache.

An Stelle jener Theorie ist bis auf die jüngsten Tage keine andere getreten, wohl aber sind Erfabrungen bekannt geworden, welche die Stellung des Vagus zu dem motorischen Apparate des Herzens viel verwickelter erscheinen lassen, als man früherhin anzunehmen Grund hatte, so lange die Beschleunigungsnerven des Herzens unbekannt waren. Nach der Entdeckung der letzteren wurden der Vagus und die Accelerantes einfach als Antagonisten angesehen, bis aus den gruindlichen Untersuchungen von $\mathrm{Bow}$ ditsch und ganz besonders ron Baxt ${ }^{1}$ ) hervorging, dass ihr gegenseitiges Verhältniss ein andersartiges sein müsse: denn ihre Gesammtwirkung bei gleichzeitiger Reizung ergab sich keineswegs als gleich der algebraischen Summe der Einzelwirkungen, wie es ein einfacher Antagonismus erfordern würde. Zeigte es sich doch z. B., dass bei gleichzeitiger maximaler Erregung des Vagus und der Accelerantes das Herz zwar zum Stillstande gebracht, also die Thätigkeit der letzteren scheinbar aufgehoben wurde, dass aber

1) N. Baxt, über die Stellung des nv, vagus zum nv. accelerans cordis. Arbeiten des physiol. Instituts zu Leipzig 1875. 
während der Vaguspause des Herzeus in seinem Mechanismus durch die Reizung der Beschleunigungsnerven Veränderungen eingeleitet wurden, welche sich nach Beendigung der Erregung beider Nerven in einer Beschleunigung der Pulsfolge von gleicher Grösse und Dauer geltend machten, als ob die nv. accelerantes für sich allein gereizt worden wären. Der Vagus ist also wohl im Stande, die Aeusserung der Accelerans-Thätigkeit für die Dauer seiner Erregung zu unterdrücken, aber nicht im Stande, den Eintritt der inneren Veränderungen zu hindern, durch deren Herbeiführung der Accelerans die Herzfrequenz steigert. Aus dieser und ähnlichen Beobachtungen scheint sich mit Nothwendigkeit zu ergeben, dass die beiderlei Nerven in dem inneren Mechanismus des Herzens nicht an dem gleichen Theile desselben in entgegengesetztem Sinne angreifen, wie es in der Voraussetzung eines einfachen Antagonismus liegen würde, sondern verschiedene Theile beeinflussen.

Bevor ich nun in der Discussion fortschreite, wird es nothwendig sein, auf die in dem nv. vagus enthaltenen Beschleunigungsfasern einzugehen, welche von vielen Seiten als Bestandtheile desselben angenommen, von andern bestritten werden. Erst wenn die Frage, ob sich vom normalen nv. vagus aus noch andere als hemmende Einwirkungen auf das Herz erzielen lassen, mit Sicherheit entschieden ist, werden die Erfolge seiner Reizung mit mehr Aussicht anf ein Verständniss derselben beurtheilt werden können.

\section{§ 4. Einwirkung des nv. vagus anf das Herz nach Nicotin- Vergiftung ${ }^{1}$ ).}

Nachdem früherhin schon von mehreren Forschern bemerkt worden war, dass nach Atropinvergiftung die Reizung des nv. vagus nicht mehr die gewohnte Verlangsamung, sondern Beschleunigung der Herzschläge zur Folge habe, ist von Sehmiedeberg ${ }^{2}$ ) Aehnliches nach Nicotinvergiftung beobachtet worden. Auf vorübergehende Reizung des Hemmungsapparates durch jenes Alcaloid folgt Lähmung, so dass der vagus nicht mehr die Pulsfrequenz zu

1) Die frühere Literatur vgl. in Auberts Bearbeitung der Innervation der Kreislauforgane. Hermanns Handbuch Bd. IV. Th. I. S. 383.

2) Schmiedeberg, Untersuchung über einige Giftwirkungen am Froschherzen. Ber, der Kön. Sächs. Gesellsch. d. Wissenschaften zu Leipzig. Mathem.-phys. Classe. 1870 S. 130. 
mindern im Stande ist. Statt der Hemmungswirkung treten bei Reizung desselben jetzt andere wichtige Erscheinungen auf: nach einer Latenzperiode von auffallend langer Dauer macht sich eine Vermehrung der Pulsschläge geltend, welche erst lange nach aufgehobener Reizung allmählich wieder weicht. Während dieser Periode der Frequenzsteigerung verlieren die Einzelpulse, mittelst eines Quecksilbermanometers aufgezeichnet, dadurch an Umfang, dass zwar die systolische Erhebung der Pulswellen an Grösse nur mässig, die diastolische Senkung aber an Tiefe bedeutend abnimmt.

Meine eigenen Beobachtungen erweitern diejenigen Schmie debergs in einigen Puncten, während sie in der Hauptsache mit denselben iubereinstimmen. Ich verwandte das Nicotin in einprocentiger Lösung, zu welcher $0,6 \%$ Kochsalz hinzugesetzt wurde. Um das ausgesehnittene Herz, während der Ventrikel seine Pulse zeichnete, zu vergiften, wurden aus einer feinen Pipette 2-3 Tropfen der Lösung auf die Vorhöfe gebracht. In der Regel dringt das Gift so allmählich ein, dass man alle Stadien seiner Einwirkung zu beobachten Gelegenheit hat. Nur wenn die letatere sehr schnell ist, sinkt die Grösse und Frequenz der Pulse rapide, bis Stillstand eintritt. Bei recht langsamer Einwirkung babe ich wiederholt die Pulse zuerst an Grösse zunehmen sehen, und zwar in der Weise, dass die diastolisehen Senkungen der Curve geringer, die systolischen Erhebungen dagegen viel grösser wurden, während keine auffällige Frequenzänderung eintrat. Dieses Stadium der Pulsverstärkung geht aber bald vorüber, um einem allmähligen Sinken der Systolegrösse bis zum schliesslichen Erlöschen der Contraction Platz zu machen. Curve VII (26. Nov. 81) giebt ein Stück eines solchen Verlaufes; bei + wurde das Gift anfgetropft. Die lange absteigende Treppe zeigt ein ähnliches Bild, wie Curve V. In anderen Fällen macht sich neben der Abnahme der Pulsgrösse eine erhebliche Verlangsamung geltend.

Was nun die Wirkung der Vagusreizung bei dem allmählichen Fortschritte der Vergiftung betrifft, so habe ich dartiber Folgendes anzugeben. Zn der Zeit, wo die Giftwirkung sich bereits in Verkleinerung der Pulsgrösse resp. Frequenz kund giebt, ist die Vagusreizung häufig von stärkerer Hemmungswirkung, als vor der Reizung, begleitet. Der Stillstand dauert selbst nach knrzer Reizung auffällig lange; nach demselben kehren die Pulse zu ihrer 
früheren Grösse und Zahl zurück. Schreitet die Giftwirkung fort, so werden nach dem durch die Reizung herbeigeführten Stillstande, welcher die Reizung kanm iuberdauert, ja selbst schon während derselben wieder aufhört, die Pulse frequenter und grösser, als vorher. Ist endlich die volle Giftwirkung eingetreten, so hat die Reizung keine Hemmung mehr zur Folge, sondern sofort eine erhebliche Frequenzvermehrung. Das letztere Stadium hat S ch mie deberg ausführlich beschrieben. Meine Curven (Vgl. C. VIII, 19. 10.81) ${ }^{1}$ ) bestätigen z. Th. die Ergebnisse von Schmiedeberg, so die auffallende Latenz und die lange Nachwirkung der Reizung, ferner die unvollkommnere Erschlaffung des Ventrikels während der Frequenzvermehrung. Sie weichen aber in einem wesentlichen Punkte von den Angaben jenes Forschers ab: die frequenteren Pulse sind gleichzeitig erheblich vergrössert, weil die systolische Erhebung erheblich wächst, während bei Schmiedeberg die systolische Erhebung sich nicht wesentlich ändert. Ich habe von diesem Verhalten nach Nicotin- (wie nach Atropin-)Vergiftung niemals eine Ausnahme gesehen; die Curven der Vagusreizung nach Einverleibung des letzteren Alcaloids gleichen vollständig der obigen Curve VIII. Der Grund der Verschiedenheit zwischen Schmiedeberg's und meinen Ergebnissen kann wohl nur in der Verschiedenheit der angewandten Registrirmethode liegen. Sc h miedeberg zeichnete mittelst eines Herzmanometers: wahrscheinlich, dass der Ventrikel sich wegen seiner stark verringerten diastolischen Ausdehnung zu wenig füllte, um bei der Systole das Manometer über die frühere Höhe heben zu können.

Auf das nicotinisirte (oder atropinisirte) Herz wirkt nach dem Voraufgehenden der Vagus genau entgegengesetzt, wie im Normalzustande: hier nimmt die Pulsfrequenz, wenn sie sich überhaupt ändert, $a b$, die diastolische Erschlaffung wird vollständiger, die systolische Contraction geringer, die Grösse der Pulse sinkt; dort nimmt die Pulsfrequenz zu, die diastolische Erschlaffung wird weniger vollkommen, die systolische Erhebung erheblich höher, die Grösse der Pulse steigt wesentlich. Wenn man bisher die Erfolge der Vagusreizung an dem durch die obigen Nareotica vergifteten Herzen auf die Erregung von Beschleunigungsfasern bezog, so ist mit dem letzteren Ausdrucke zu wenig gesagt, denn es handelt

1) Die Reizung ist auf der untersten Linie durch die Pfeile $\nearrow \nwarrow$ bezeichnet. 
sich nicht bloss um Steigerung der Pulsfrequenz, sondern ebenso sehr auch um Steigerung der Pulsgrösse. Ob aber der Gegensatz der Vaguswirkung am normalen und am vergifteten Herzen auf der Erregung zweier verschiedener Faserclassen beruht, ist nach den bisher mitgetheilten Thatsachen nicht mit Sicherheit zu entscheiden. So einfach und naheliegend diese Annahme auch ist, es wäre doch nicht undenkbar, dass die Alcaloide die Erregbarkeit eines einheitlichen nervösen Apparates der Art umstimmten, dass sich seine normale Wirkungsweise in die gegentheilige verkehrte. Eine Entscheidung dieser Frage wird erst nach Sammlung weiterer Erfahrungen möglich sein.

Nach Angaben von Schelske ${ }^{1}$ ), welche trotz vielseitiger Widersprïche noch immer wiederholt werden, soll der vagus auf ein auf $32^{\circ} \mathrm{C}$. erwärmtes Froschherz motorisch einwirkeu. Ich habe eine grosse Zahl von Versuchen angestellt, indem ich das Herz auf ein Kästchen mit Warmwasser-Heizung legte. So interessant derartige Beobachtungen in rieler Beziehung; z. B. rûcksichtlich des Einflusses der Temperatursteigerung auf die Frequenz und den zeitlichen Verlauf der Ventrikelcontractionen sind - ich habe nie eine andere als die gewöhnliche Hemmungswirkung gesehen, wie es Ludwig und Luchsinger ${ }^{2}$ ) ebenfalls ergangen ist. Ebenso theile ich mit den letzteren Autoren das Schicksal, an Herzen von "Salzfröschen" nur die gewöhnliche Vaguswirkung beobachtet zu haben, im Gegensatz zu Sehiff, welcher den Vagus auf das entblutete Herz beschleunigend einwirken sah.

\section{§. Verstärkung der Thätigkeit des normalen Herzens durch electrische Vagusreiznng.}

Dass durch electrische Reizung des nv, vagus von einer gewissen Stärke Beschleunigung des Herzschlages herbeigefuihrt werden könne, ist bekanntlich eine viele Jahre hindurch von Sch iff und von Moleschott vertheidigte, von andern Seiten mit Entschiedenheit bestrittene Behauptung.

In seltenen, aber von jedem Verdachte eines Irrthums freien Fällen habe ich am normalen Herzen durch electrische Reizung des nv. vagus Curven erhalten, welche vollständig der am Nico-

1) R. Schelske, über die Veränderungen der Erregbarkeit der Nerven durch die Wärme. Habilitationsschrift. Heidelberg 1860.

2) Ludwig und Luchsinger, Pfl̈̈gers Archiv Bd. XXV. S. 217. 
tinherzen gewonnenen Curve VIII glichen: der Reizerfolg bestand also in Steigerung der Pulsfrequenz, erheblicher Vergrösserung der systolischen Erhebungen und merklicher Abnahme der diastolischen Senkungen. Weshalb es mir nur so selten gelungen ist, auf dem Wege electrischer Reizung diese Wirkung zu erzielen, vermag ich nicht anzugeben.

Dagegen erhält man an fast jedem noch nicht ermideten Herzen als Nachwirkung dër electrischen Reizung, welche während ihrer Dauer Herzstillstand bedingt, Beschleunigung und Vergrösserung der Pulse unter der Bedingung, dass man zu der Reizung hinreichend starke Ströme wählt.

Die Beobachtung, dass nach vorgängigem durch Vagusreizung erzwungenem Stillstande die Pulse frequenter und kräftiger werden können, ist schon hier und da gemacht ${ }^{1}$ ), aber nicht die Bedingung: ermittelt worden, unter welcher dieser Erfolgg eintritt. Man hat wohl gemeint, dass während des Herzstillstandes in den motorischen Ganglienzellen des Herzens Spannkräfte aufgespeichert würden, welche beim Wiederbeginne der Pulsationen zu ungewöhnlich kräftiger Erregung der motorischen Nerven und dadurch der Herzmuskulatur führen, bis der angesammelte Vorrath erschöpft sei.

Indess lässt sich zeigen, dass diese Auffassung nicht stichhaltig ist. Wäre sie richtig, so maïsste die Grösse der Nachwirkung von der Dauer des Herzstillstandes abhängig sein, denn mit dieser müsste ja die Summe der sich ansammelnden Spannkräfte bis zu einer gewissen Grenze zunehmen.

Allein wenn man durch Reizung desselben vagus mittelst schwächerer und stärkerer Ströme gleich lange Herzpausen erzielt, ist die Nachwirkung der stärkeren Ströme auf die Frequenz und Grösse der Ventrikelcontractionen trotz der gleichen Dauer des Stillstandes viel erheblicher, als die Nachwirkung der schwächeren Ströme. Ja es gelingt nicht selten, schwache Ströme innerhalb solcher Intensitätsgrenzen zu finden, dass die Pulse nach dem Stillstande nur zu der ursprünglichen Grösse und Frequenz zurückkehren, während eine unmittelbar darauf folgende stärkere Reizung bei gleichem oder selbst kürzerem Stillstande eine nachträgliche Verstärkung der Ventrikelcontractionen vori erheblichem Umfange und erheblicher Dauer hinterlässt.

1) Vgl. z. B. Schiff, Arch. f. physiol. Heilk. VIII. 183. 1849. Ludwig und Hoffa, Henle und Pfeuffers Zeitschrift Bd. 9. 1850. S. 107. 
Untersuchungen über den Einfluss des nv. vagus auf die Herzthätigkeit. 397

Curve IX $a$ und $b$ mögen diese Verhältnisse erläutern. In Curve IX a wurde der Vagus bei dem Schlittenstande 80-65 so lange tetanisirt, wie es die beiden Pfeile auf der untersten Linie angeben. Nach dem Stillstande erreichen die Ventrikelcontractionen nur die urspringliche Energie und Zahl. In Curve b. wurde viel kürzere Zeit bei dem Schlittenstande 15 tetanisirt: die Pulse steigen trotz der kürzeren Dauer der Herzruhe weit über die Anfangswerthe der Grösse und Frequenz hinaus und kehren nur sehr allmählich zu jenen zurück. In beiden Curven tritt die starke Erschlaffung des Ventrikels wäbrend des Stillstandes, in Curve IX b die Beschränkung der diastolischen Senkung während der Nachwirkung auf das deutlichste hervor.

Es kann mithin keine Rede davon sein, dass die positive Nachwirkung der Vagusreizung - so will ich der Kürze halber die nachträgliche Steigerung der Frequenz und Höhe der Pulsationen nennen - durch die voranfgehende Herzruhe an sich bedingt sei. Es muss vielmehr neben der Hemmungswirkung eine zweite Wirkung einhergehen, die erst bei stärkerer Reizung eintritt und deren Aeusserung durch die Hemmungswirkung für die Dauer der letzteren unterdrückt wird. Die positive Nachwirkung klingt im Laufe der Zeit in ganz ähnlicher Weise ab, wie die Verstärkung der Herzpulse nach Vagusreizung am nicotinisirten Herzen: in beiden Fällen sinken Grösse und Frequenz der Ventrikelcontractionen nur sehr allmählich auf die vor der Reizung bestandenen Werthe herunter. Der Verlauf der Curve IX $b$ hat die vollständigste Analogie mit den Frequenzeurven, welche Baxt durch gleichzeitige Reizung des Vagus und der Accelerantes am Säugethierherzen gewann.

Es giebt aber noch andre interessante Fälle positiver Nachwirkung. Bei Froschherzen, welche bereits längere Zeit ausserhalb des Körpers pulsirt haben, tritt nicht selten eine Periode ein, in welcher die Ventrikelpulse aussetzen, der Art, dass nur auf jeden zweiten oder dritten Vorhofsschlag eine Zusammenziehung des Ventrikels erfolgt. Unter diesen Bedingungen sieht man oft nach vorausgegangener Vagusreizung die Pulsfolge wieder regelmässig werden, gleichviel ob die Reizung Herzstillstand zur Folge hatte oder der letztere durch Atropin verhindert worden war. Curve X (25. October 1881) giebt ein Beispiel der Art von einem atropinisirten Herzen. Das Plättchen des Zeichenhebels ist gleichzeitig auf Vorhof 
und Ventrikel gesetzt. Wo der Vorhof allein schlägt, wird eine kleine Welle gezeichnet, wo auf den Vorhofspuls ein Ventrikelpuls folgt, wird die dem ersteren entsprechende kleine Welle durch die viel grössere, der Ventrikelcontraction entsprechende Erhebung: verdeckt. Vor der Reizung setzt der Ventrikel regelmässig ein Mal aus, nach derselben werden die Ventrikelpulse für eine Zeit lang regelmässig, bis wieder zuerst der dritte, dann der zweite Schlag ausfällt ${ }^{1}$ ). - In anderen Fällen setzt der Ventrikel zwar nicht aus, macht aber abwechselnd kleine und grosse Schläge; nach Vagusreizung werden sie für eine Zeit lang durch Vergrösserung der kleineren gleich hoch. -

Die Gesammtheit der in dem vorliegenden und dem voraufgehenden Paragraphen mitgetheilten Thatsachen lassen darüber keinen Zweifel, dass der Vagus auf das Herz zweierlei Wirkungen entgegengesetzter Art ausuuüben im Stande ist, die früherhin nur unvollständig durch Verlangsamung oder Beschleunigung der Pulse charakterisirt worden sind. Die eine Wirkung äussert sich in Verringerung der Zahl und Grösse der Pulse, sowie in Beförderung der diastolischen Erschlaffung, die andre in Steigerung der Zahl und Grösse der Pulse, wie in Beeinträchtigung der diastolischen Erschlaffung. Die zweite Wirkung tritt theils als primäre, theils als Nachwirkung der electrischen Reizung auf: als primäre in seltenen Fällen am normalen, regelmässig am nicotinisirten oder atropinisirten Herzen, als Nachwirkung nach voraufgegangenem Stillstande bei starker Vagusreizung.

Gegen alle diese Versuche erhebt sich nun aber ein gewichtiger Einwand. Schon behufs Herbeiführung des Herzstillstandes erfordert der nv. vagus des Froschherzens ziemlich starke Ströme, vollenids behufs Erzielung einer positiven Nachwirkung. Trügerische Stromschleifen oder unipolare Abgleichungen, das Herz unmittelbar treffend, könnten leicht die positive Nachwirkung als Folge der Vagusreizung vorspiegeln, während in Wirklichkeit eine Folge directer electrischer Herzreizung vorliegt.

Ich brauche kaum zu erwähnen, dass ich keine der ïblichen Controllmaassregeln versäumt habe, um mich gegen derartige Täuschungen sicher zu stellen. Durchschneidung des Nerven unter-

1) Früherhin hat ähnliche Beobachtungen schon Coats, neuerdings Gaske11 gemacht. 
Untersuchungen über den Einfluss des nv. vagus auf die Herzthätigkeit. 399

halb der Electroden und Wiederverklebung der Schnittflächen, Unterbindung mit feuchtem Faden heben die Reizerfolge aufi. Aber diese Ueberwachungsmethoden ändern unläugbar auch den Leitungswiderstand unterhalb der Electroden und geben deshalb keine absolute Garantie. Stromprüfende Froschschenkel, mit dem Nerven in den verschiedensten Richtungen uiber das Herz gelegt, blieben bei einer grossen Zahl von Versuchen vollständig ruhig. Es war interessant, dass in einer Reihe von Fällen um die Zeit der positiven Nachwirkung der Vagusreizung secundäre Zuckung eintrat, die vorher fehlte, - ein neues Zeichen der Verstärkung der Ventrikelcontractionen w.ährend jener Periode. Jene negativen Erfahrungen hatten mich bereits über die Zutrauenswürdigkeit meiner Versuche beruhigt, als in zwei Fällen unter allerdings sehr vielen dennoch Tetanus des Schenkels beim Tetanisiren des Vagus eintrat. Demnach war wenigstens die Möglichkeit einer directen electrischen Herzreizung bei meinen Anordnungen dargethan und die Sicherung der Ergebnisse durch anderweitige Reizmethoden geboten.

\section{§. 6. Chemische Reizung des nv. vagus.}

Das Mittel dazu fand sich bald in der Anwendung concentrirter Kochsalzlösung auf den Nerven. Concentrirtes Glycerin erwies sich weniger constant im Erfolge, als Chlornatrium. Der nv. vagus des auf dem Schreibeapparate angebrachten Herzens wurde an einen Seidenfaden geknïpft und mittelst desselben auf einem Glasplättchen so fixirt, dass zwischen dem Rande des letzteren und dem Herzen der Nerv sich frei ausspannte. Obne diese Anordnung fliesst ein Tropfen Kochsalzlösung, welcher auf dem Plättchen in Berührung mit dem Nerven gebracht wird, leicht auf das Herz selbst über. Der Erfolg der Kochsalzreizung kann nun ein mehrfacher sein.

1) Die erste, einige Zeit nach Application der Salzlösung eintretende Wirkung besteht in Hemmung der Herzthätigkeit, welche sich entweder nur in Verringerung der Frequenz und Höhe der Pulse oder in Herbeiführung längeren Stillstandes äussert. Durchschneidet man den Nerven, nachdem die Hemmung einige Zeit bestanden, dicht vor dem Herzen, so tritt starke positive Nachwirkung, ganz wie bei electrischer Reizung des vagus ein. Vgl. Curve XI (28. 12. 81.): das Stiick e d schliesst unmittelbar an a b 
an. Bei $+S$ wird Salzlösung auf den Nerven gebracht. Nach einiger Zeit sinkt die Frequenz und Höhe der Pulse, die diastolische Erschlaffung wird bedeutender, Bei $+d$ Durchschneidung des Nerven, gefolgt von sehr erheblicher, allmählich abklingender positiver Nachwirkung.

Wenn man den Nerven bei bestehender Hemmungswirkung nicht durchschneidet und dafür sorgt, dass die Salzlösung nicht an demselben entlang kriecht, sieht man nach längerer Zeit die Hemmungswirkung nachlassen und eine positive Wirkung anftreten, die aber nicht bloss als Nachwirkung aufzufassen ist. Denn sie sinkt sofort, wenn während ihres Bestehens der Nerv durchtrennt wird, zum Zeichen, dass sie von einer an der benetzten Stelle des Nerven noch bestehenden Reizung abhängt. In der ersten Zeit der Kochsalzeinwirkung tritt also die Hemmungs-, in der spätern die Verstärkungswirkung in den Vordergrund. Curve XII (31. 12. 81.) zeigt dies Verhalten. Die einzelnen über einander stehenden Stücke schliessen in der Reihenfolge ab-cd-ef an. Bei $+\mathrm{k}$ wird ein Kochsalzkrystall auf den Nerven gebracht. Er bewirkt zuerst Verringerung der Pulshöhe und Frequenz; die Contractionen steigen aber mit der Zeit wieder an and weit über die ursprüngliche Grösse hinaus, um nach der Durchschneidung (bei $+d$ ) sofort wieder abzusinken.

2) In der eben besprochenen Curve sieht man (an der mit! bezeichneten Stelle bei $\alpha$ ) vor Eintritt der Hemmung die Pulse eine kurze Zeit hindurch an Grösse zunehmen. Dies Verhalten bildet den Uebergang zu einem andern, in der Curve XIII (20. Dec. 81) dargestellten: Bei $+\mathrm{S}$ wird die Salzlösung auf den Nerven gebracht; nach einiger Zeit tritt ohne vorherige Hemmung erhebliche positive Wirkung auf, d. h. Beschleunigung und Vergrösserung der Pulse. Bei d Durchschneidung, die (s. den oberen Theil der Curve) sehr bald Absinken der Curvengipfel herbeiführt.

In nicht seltenen Fällen kommt es bei der chemischen Reizung vor, dass die Herzcontractionen sich zwar erheblich verstärken, aber nicht gleichzeitig beschleunigen, wovon Curve XIV (19.12. 81) ein gutes Beispiel giebt. Das Salz war auf den Nerven schon 210 Sec. vor dem Anfange des hier abgebildeten Curvenstückes aufgetragen, ohne die Herzpulse irgend wie zu ändern. Man sieht, wie einige Zeit nach Beginn des Curvenabschnittes die Pulse um Erhebliches wachsen, ohne ibre Frequenz zu ändern. Nach der 
bei + erfolgten Durchschneidung sinken sie schnell, zum Zeichen, dass die Verstärkung wirklich von der Reizung herrührte.

Die Kochsalzreizung beweist nach den vorgelegten Resultaten mit Sicherheit, dass der gegen die electrische Reizung erhobene Verdacht unbegründet war, denn sie bestätigt durchans Alles, was die letztere gelehrt hat: die Doppelwirkung der Vagusreizung ist nunmehr über allen Zweifel festgestellt.

Der Fall, dass positive Wirkung der Reizung ohne vorgängige Hemmung auftritt, war bei der Verwendung electriseher Ströme am normalen Herzen ein im Ganzen seltener; bei Verwendung der Kochsalzlösung ist er sehr häufig. Woher dieser Unterschied, weiss ich nicht mit Sicherheit zu sagen, ebenso wenig, weshalb die positive Wirkung bald als primäre, bald als nachträgliche auftritt. Von der "Individualität" der verschiedenen Herzen hängt dies nicht ab, denn auf dasselbe Herz wirkt oft der eine Vagus, durch Kochsalz gereizt, primär hemmend, der andere primär erregend. Es liegt die Vermutbung sehr nahe, dass die räumliche Anordnung der Fasern in dem Nervenstamme und das allmähliche Eindringen der Salzlösung in denselben, bei welchem bald die einen bald die andern Fasern zuerst gereizt werden, an diesem Schwanken des Verhaltens Schuld trägt.

§. 7. Hängt die hemmende und die verstärkende Wirkung des Vagus auf die Herzthätigkeit von den gleichen oder von verschiedenen Nervenfasern ab?

Nachdem die erste Frage (vgl. $\S 1$ ), in deren Interesse die vorliegende Untersuchung unternommen wurde, nämlich, ob dem nv. vagus neben seiner unbestrittenen hemmenden auch eine, oft bestrittene, erregende Einwirkung auf das Herz zukomme, in positivem Sinne mit aller Sicherheit entschieden worden, gilt es nun, auch die zweite Frage zu beantworten, ob beiderlei Einwirkungen von den gleichen oder von verschiedenen im Vagusstamme enthaltenen anatomischen Elementen abhängen. Ich habe mich früherhin der ersteren Annahme zugeneigt ${ }^{1}$ ), aber dieser Vermuthung die Bemerkung hinzugefügt, dass es Nerven gebe, die ohne Zweifel nur hemmend, niemals erregend wirken, (z. B. die chorda tympani

1) R. Heidenhain, über Erregung und Hemmung. Pfiügers Archiv Bd, 26. S. 556 น. 557. 1881. 
bezïglich der Gefässe der Speicheldrïsen, der nv. depressor bezüglich des vasomotorischen Centrums u. s. f.) und dass solche Fälle schlechterdings einsinniger Wirkung doch immer wieder die Erwägung nahe legen, ob bei doppelsinniger Wirkung trotz der anscheinenden Möglichkeit, dieselbe auf die gleichen Elemente unter verschiedenen Bedingungen ihrer Thätigkeit zu beziehen, dennoch zweierlei entgegengesetzt wirkende Classen von Nervenfasern vorhanden seien.

Seit man an dem Herzagus gelernt hat, theils durch Gifte, theils durch vorgängige Durchschneidung ${ }^{1}$ ) seine Hemmungswirkung aufzuheben und die Beschleunigungswirkung hervortreten zu lassen, ist die Annahme besondrer beschleunigender neben den hemmenden Fasern eine allgemeine geworden. Nothwendig scheint sie indessen nach jenen Versuchen nicht; denn das Gift in dem einen, in dem andern Falle die eintretende Degeneration der Fasern könnten eine Aenderung der Erregbarkeit in dem motorischen Apparate des Herzens hervorrufen, welehe die normale Hemmungswirkang des Vagus in ihr Gegentheil verkehrt. Sieht man doch, wie Cyon gefunden und ich für Kaninchen bestätigt habe, die Reizung sensibler Nervenstämrne nach Chloralinjection in das Blut nicht, wie unter normalen Verhältnissen, von Steigerung, sondern von Herabsetzung. des Blutdruckes begleitet; es wird aber wohl kaum Jemand annehmen wollen, dass jeder sensible Nerv besondere pressorische und besondere depressorische Fasern enthalte, vielmehr Jedermann diese Umkehr der Wirkung auf Veränderung der Erregbarkeit der Centraltheile zu beziehen geneigt sein. Das Herz betreffend, so hat jüngst $\mathrm{H}$. Munk für den intracardialen Theil seines Nervensystems die Identität hemmender und erregender Fasern behauptet, und ganz neuerdings ist Gaskell in dem mehrfach citirten Auszuge seiner in Aussicht gestellten Abhandlung gegen eine Vielfachheit der Herzfasern des Vagus aufgetreten.

Nachdem ich mich mehrere Monate mit der in Rede stehenden Frage beschäftigt, kann ich nicht mehr daran zweifeln, dass der Hemmung und der Verstärkung der Herzthätigkeit zwei verschiedene Classen von Nervenfasern entsprechen. Die Grïnde sind

1) M. Schiff, über den Ursprung der erregenden Herznerven. Pflügers Archiv Bd. XVIII. O. 172. 1878. - F. Klug, über die Beschleunigungsnerven des Froschherzens. Med. Centralbl. 1881. No. 53. 
Untersuchungen über den Einfluss des nv. vagus auf die Herzthätigkeit. 403

theils in den bisher mitgetheilten, theils in noch weiteren, später zu besprechenden Thatsachen enthalten. -

Hinreichend starke electrische oder chemische Reizung des Vagusstammes bedingt, wie gezeigt worden ist, zwei entgegengesetzte Erfolge am Herzen: Stillstand während der Dauer, Verstärkung der Herzthätigkeit (Vergrösserung der Zahl und Höhe der Pulse) nach Schluss der Reizung. Leztere Wirkung ist nicht unmittelbare Folge der ersteren, denn sie folgt nicht auf einen durch hinreichend schwache Ströme, sondern nur auf einen durch starke Ströme herbeigeführten Herzstillstand, wenn letzterer auch kürrzere Zeit dauert als ersterer; - sie ist vielmehr eine zweite, neben der Hemmung sich gestaltende Einwirkung auf das Herz, deren Aeusserung nur durch die Hemmung unterdrückt, deren Entwicklung aber durch dieselbe nicht aufgehoben wird. Es ist, glaube ich, schlechterdings die Möglichkeit nicht abzusehen, die beiderlei gleichzeitig eingeleiteten entgegengesetzten Wirkungen von ein und derselben Faserart abzuleiten.

Doch wünschte ich die Existenz zweier verschiedener Faserclassen noch directer darzuthun, dureh anatomische Isolirung derselben, was mir bis zu einem gewissen Grade auch gelungen ist. -

Der erste Schritt in dieser Ricbtung bezeichnet freilich einen Misserfolg. Ich wandte mich an das Herz, um eine Beobachtung von Eckhard möglicher Weise für meine Zwecke weiter zu verwerthen. Nach diesem Forscher soll der Vagus seine hemmende Wirkung noch beibehalten, nachdem beide Scheidewandnerven durchtrennt worden sind ${ }^{1}$ ). Ich wollte nun zusehen, $a b$ nach dieser Operation auch die verstärkende Einwirkung des Vagus auf die Herzthätigkeit fortbestände oder ob sie fortfiele. Im letzteren Falle würde die Verschiedenheit der hemmenden und der verstärkenden Fasern nachgewiesen sein. Ich habe nun den Eckhard'schen Versuch an einer grossen Zahl von Fröschen ausgeführt, aber zur Reizung des nv. vagus nicht electrische Ströme, sondern Kochsalzlösung angewandt, welche bei intactem Herzen unfehlbar die oben besprochenen Wirkungen zeigt. Nach Durchtrennung der Scheidewandnerven, deren Gelingen ich stets microscopisch constatirte, fiel jeder Einfluss der Kochsalzreizung des vagus auf das Herz

1) Eckhard, Beiträge zur Anatomie und Physiologie Bd. VII. S. 190. 1876. 
fort. Je unerwarteter mir gegenüber Eckhard's Erfahrungen dies negative Ergebniss war, desto mehr habe ich mich vor Versuchsfehlern zu schützen gestrebt. In einer Anzahl von Fällen reizte ich zuerst den einen Vagus, um mich des positiven Erfolges zu versichern, trennte dann die Scheidewandnerven and reizte nunmehr in der frühern Weise den andern Vagus. Der Erfolg blieb stets und ohne Ausnahme negativ; ich musste deshalb von diesem Versuchswege für meine Zwecke absehn.

Demnächst schritt ich zur Reizung der medulla oblongata. Electrische Ströme sind hier nicht anwendbar, da zu den Nervenstämmen vordringende Stromschleifen kaum auszuschliessen sein dürften; ich verwandte deshalb als Reizmittel nur Kochsalz. Die medulla wurde von hinten freigelegt und ein Tropfen concentrirter Salzlösung auf den hintersten Theil des Bodens der Rautengrube gebracht, oder sie wurde von vorne aufgedeckt und Querschnitte dicht über oder dicht unter dem Niveau der Vaguswurzeln oder auch ein medianer Längsschnitt angelegt und diese Schnittflächen mit Salzlösung in Berührung gebracht. Das Herz hing nur dureh die beiderseitigen Vagi mit den Centraltheilen zusammen. Derartige an 42 Fröschen angestellte Versuche gaben folgende Resultate:

1) Primäre Verstärkung der Herzthätigkeit, wie sie bei Salzreizung der vagi sehr häufig anftritt, kam nie zur Beobachtung.

2) Hemmung tritt stets auf, in der Regel so energisch, dass das Herz lange stillsteht.

3) Wenn man, nachdem der Stillstand längere Zeit gewährt hat, die Vagi durchtrennt, treten schnell wieder Pulse ein. In 32 von jenen 42 Fällen ging ihre Frequenz und Höhe auf den ursprünglichen Werth zuruick oder erreichte diesen nicht. In nur 10 Fällen wuchs die Höhe der Pulse über den Anfangswerth, es trat also positive Nachwirkung auf, aber nie in so erheblichem Grade, wie sie in der Regel bei chemischer Reizung des Vagus-Stammes stattfindet.

4) Unterlässt man die Nerventrennung während des Herzstillstandes, so bleiben die mit der Zeit wiederkehrenden Pulse fast immer an Frequenz und Höhe hinter den ursprünglichen zurtick, während sie unter ähnlichen Bedingungen bei Reizung des Nerrenstammes fast immer über dieselben hinausgehn.

Vergleicht man die Erfolge der Kochsalzreizung der medulla 
und des nv. vagus selbst, so gelangt man zu interessanten Schlussfolgerungen. Zunächst bestätigt sich, was schon oben erörtert worden ist, dass die positive Nachwirkung bei Reizung des Nervenstammes nicht durch die voraufgehende Herzruhe bedingt ist. Denn bei Reizung der medulla tritt nach langer Herzpause nur in seltenen Fällen positive Nachwirkung auf, letztere kann also nicht nothwendige Folge der ersteren sein. Die Momente, welche die Nachwirkung bei Reizung des Nervenstammes so regelmässig auftreten lassen, muissen bei Reizung der medulla nur selten zur Wirksamkeit gelangen. An was anders kann man dabei wohl denken, als an besondre, von den hemmenden verschiedne Fasern innerhalb des Stammes?

Es fehlen jene den Herzschlag verstärkenden Elemente in der medulla nicht, denn in einer gewissen Zahl von Fällen kommt die positive Nachwirkung zur Beobachtung. Dass dies aber so selten geschieht, kann, so weit ich sehe, auf nichts Anderem beruhen, als auf einer besondern räumlichen Disposition der hemmenden Elemente einerseits, der verstärkenden andrerseits. In dem Stamme sind beiderlei Elemente gesammelt, in der medulla scheinen die hemmenden ebenfalls räumlich zusammengedrängt zu sein, vermuthlich in dem Vagus-Kerne, so dass sie von dem chemischen Reizmittel gleichzeitig in grösserer Zahl afficirt werden, während die verstärkenden Elemente räumlich mehr zerstreut sind, so dass der chemische Reiz schwieriger eine' grössere Zahl derselben gleichzeitig trifft und deshalb schwieriger zu so energischer Wirksamkeit gelangt, wie in dem Stamme. Daher tritt auch niemals Verstärkung der Herzthätigkeit als primäre Wirkung der chemischen Markreizung auf: die in grösserer Zahl erregten hemmenden Elemente behalten zunächst stets die Oberhand.

Die vorliegenden Thatsachen führen mithin zu der früherhin allgemein getheilten, neuerdings von manchen Seiten her erschuitterten Ueberzeugung, dass die Hemmungs- und Verstärkungswirkung: des Vagus von zwei verschiedenen, in seinem Stamme gleichzeitig: enthaltenen Faserclassen abhängt. Die verstärkenden Fasern sind die vollständigen Analoga der nervi accelerantes des Sängethierherzens. Denn 1) beiderlei Fasern beeinflussen die Herzthätigkeit, wenn erregt, erst nach einem auffallend langen Stadio der Latenz; 2) beide hinterlassen im Herzen nach Beendigung ihrer Reizung: eine auffallend lange und sehr langsam abnehmende Nachwirkung. 
3) Beide werden zwar durch gleichzeitige Reizung der Hemmungsfasern in der Aeusserung ihrer Thätigkeit gehindert, aber nicht in der Einleitung derjenigen Veränderungen im Herzen, welche zu einer Verstärkung seiner Thätigkeit führen, sobald die Reizung der Hemmungsfasern ihr Ende erreicht hat.

Nur in einem Punkte scheint beim ersten Anblicke die Einwirkung der Verstärkungsfasern auf das Froschherz von der Einwirkung der nv. accelerantes auf das Säugethierherz verschieden zu sein. Alle Beobachter, welche die Wirkung der letzteren auf den Herzschlag mittelst eines Arterien-Manometers darstellten, sahen die Pulse an Frequenz zunehmen, aber an Grösse erbeblich sinken, während die Contractionen des Froschventrikels gleichzeitig mit der Frequenz- auch eine erhebliche Grössezunahme zeigen. Diese Verschiedenheit beruht aber wohl nur auf der Verschiedenheit der Beobachtungsmittel. Es hat sich, in Uebereinstimmung mit den Beobachtungen Schmiedebergs am nicotinisirten, bei meinen Curven auch am unvergifteten Herzen gezeigt, dass die Reizung der Verstärkungsnerven die diastolische Ausdehnung des Ventrikels beeinträchtigt. Wenn sich aber der Ventrikel weniger ausdehnt, muss die Blutmenge, welche er bei der Systole in die Arterien wirft, sinken und damit nothwendig die rom ArterienManometer gezeichnete Pulswelle an Grösse abnehmen. Die unmittelbare graphische Darstellung der Herzcontractionen lehrt in der Verstärkung der Ventrikelsystole eine Folge der Reizung der Verstärkungsnerven kennen, welche sich in den mittelst des $\mathrm{Ar}^{-}$ terienmanometers gezeichneten Pulscurven nicht ausdrücken konnte.

Wenn ich annehme, dass nicht bloss die Hemmungsfasern, sondern anch die Verstärkungsfasern des vagus aus der medulla stammen, so trete ich damit in Widerspruch zu einer neuerdings ausgesprochenen Vermuthung F. Klugs's). Dieser Forscher sah einige Wochen nach Durchschneidung des ram. intestinalis nv. vagi bei Reizung desselben den Herzschlag sich beschleunigen oder das schon stillstehende Herz wieder in Thätigkeit gerathen. Die Hemmungsfasern waren also unerregbar geworden, die Verstärkungsfasern waren noch erregbar. Klug meint nun, dass der Ursprung der letzteren in den von ihm im Verlaufe der Nerven auf-

1) F. Klug, über die Beschleunigungsnerven des Froschherzens. Medic. Centralbl. 1881. No. 53. 
gefundenen Ganglienzellen zu suchen sei. Allein dann würden sich die Fälle nicht erklären, in denen ich von dem verlängerten Marke aus positive Nachwirkung erhielt. Ueberdies lässt sich die Bedeutung von Beschleunigungsfasern schwer verstehen, welche aus peripherisch gelegenen unipolaren Ganglienzellen stammen und keine centrale Verbindung haben; unter welchen physiologischen Bedingungen sollten sie wohl in Erregung gerathen? Denkbar wäre es freilich, dass von dem obersten sympathischen Ganglion aus Beschleunigungsfasern in das Vagus-Ganglion einträten, welches mit jenem durch starke Anastomosen verbunden ist. Solche Fasern könnten von andern sympathischen Verbreitungrsbezirken her auf der Vagusbahn Erregungen zum Herzen leiten. Dank der überaus grossen Gefälligkeit des Herrn Klug, der mit liebenswürdigster Bereitwilligkeit das hiesige Institut mit den herrlichen Fröschen aus der Umgebung Klausenburgs versorgt, war ich im Stande, an einer Anzahl grosser ungarischer Frösche Reizversuche an dem sympathischen Ganglion mit Kochsalz anzustellen, die bei unsern kleinen Fröschen schwer möglich sein würden, aber ich habe niemals eine Veränderung des Herzschlages von dorther erzielt. So bleibt denn, so weit ich sehe, kaum ein Ausweg, als die medulla als alleinigen Ursprungsort auch für die Verstärkungsfasern anzusehen. Dass sie, wie Schiff beim Hunde und Klug beim Frosche gesehen, nach Durchschneidung der Nerven ihre Erregbarkeit länger behalten, als die Hemmungsfasern, steht nicht ausser Analogie. Wissen wir doch, dass nach Durchschneidung des nv. ischiadicus beim Hunde die Erweiterungsfasern für die Gefässe der Pfote länger erregbar bleiben, als die Verengerungsfasern; es können also Fasern desselben Nervenstammes von ungleicher physiologischer Bedeutung nach Trennung des Stammes ungleich schnell ihre Erregbarkeit einbiissen. Klugs Beobachtungen scheinen mir demach den centralen Ursprung der Verstärkungsfasern nicht zu widerlegen.

\section{\& 8. Schlussbemerkungen.}

Es ist also kein Zweifel, der nv. vagus enthält in der That zwei verschiedene Faserclassen für das Herz. Die Wirkung der einen besteht in Herabsetzung der Pulsgrösse, der Pulsfrequenz 
und in Vergrösserung der diastolischen Erschlaffung des Herzens, die Wirkung der andern in Steigerung der Pulsgrösse, der Pulsfrequenz und Verringerung der diastolischen Erschlaffung. Die bisher iibliche Bezeichnung der letzteren als „Beschleunigungsfasern" erscheint nicht ausreichend, da die Beschleunigung der Herzschläge nur eine Seite ihrer Einwirkung auf das Herz darstellt; die Bezeichnung als „Verstärkungsfasern“ erscheint zutreffender.

Man könnte geneigt sein, die Zunahme der diastolischen Erschlaffung bei Reizung der Hemmungsfasern, ihre Abnahme bei Reizung der Beschleunigungsfasern, nur in Zusammenhang mit den jene Reizungen begleitenden Aenderungen der Pulsfrequenz zu bringen; je langsamer das Herz schlägt, je länger die Diastole danert, desto mehr haben die systolisch zusammengezogenen Muskelfasern wieder Zeit sich auszudehnen und umgekehrt. Allein ein solcher nnmittelbarer und nothwendiger Zusammenhang zwischen Pulsfrequenz und diastolischer Erschlaffung, wonach letztere im umgekehrten Verhältnisse zu ersterer stände, besteht in Wirklichkeit nicht. Es giebt Bedingungen, unter denen die Frequenz sehr hoch und die diastolische Ausdehnung sehr vollkommen ist, andere, unter denen die Frequenz viel geringer und dennoch die diastolische Ausdehnung sehr viel weniger vollkommen ist. Ein Beispiel der Art liefert Curve XV (8. Nov. 81.), welche sich auf die Herzschläge bei versehiedenen Temperaturen bezieht. Bei $31^{\circ} \mathrm{C}$. war die Frequenz sehr hoch, die Erschlaffung sehr vollständig, bei allmählich auf $9^{\circ} \mathrm{C}$. sinkender Temperatur nahmen beide Grössen gleichzeitig erheblich ab.

Steigerung der Frequenz bedingt also nicht an sich Verringerung der diastolischen Ausdehnung. Wo letztere neben der ersteren vorkommt, muss sie einen besonderen, in den innern Zuständen des Herzmuskels gelegenen Grund haben.

Aus dem Umstande, dass der Umfang, welchen die diastolische Erschlaffung unter gewöhnlichen Umständen zeigt, durch Reizung der Hemmungs- und Verstärkungsfasern vergrössert und verkleinert werden kann, folgt mit Nothwendigkeit, dass für gewöhnlieh der Ventrikel während der Diastole nicht vollständig erschlafft, sondern bis zu einem gewissen Grade contrahirt bleibt und dass der Grad dieser diastolischen Zusammenziehung unter verschiedenen Umständen erheblicher Schwankungen fähig ist. 
Untersuchungen über den Einfluss des nv. vagus auf die Herzthätigkeit. 409

Gaske I1 1) hat den Grad der diastolischen Ausdehnung (oder Zusammenziehung) des Herzens mittelst des Roy'schen Tonometers unter verschiedenen Umständen untersucht und gefunden, dass Alkalien, Antiarin, Digitalin die diastolisehe Ausdehnung des Herzens verringern, während die systolische Zusammenziehung ihrem Grade nach dieselbe bleibt, aber ihre Dauer anwächst. Bei Einwirkung von Säuren umgekehrt bleibt die diastolische Erschlaffung gleich, die systolische Zusammenziehung aber nimmt mehr und mehr ab. Es würde verführerisch sein, die Einwirkung der Hemmungsfasern mit der von Säuren, die Einwirkung der Verstärkungsfasern mit der von Alkalien zu vergleichen, wenn nicht die Verstärkungsfasern mit der Verringerung der diastolischen Ausdehnung eine bedeutende Verstärkung der systolischen Zusammenziehung veranlassten, die bei der Einwirkung von Alkalien ungeändert bleibt, und wenn nicht die Hemmungsfasern gleichzeitig mit der Herabsetzung der systolischen Contraction eine Vergrösserung der diastolischen Erschlaffung herbeiführten, welche die Säuren nicht bewirken. Will man die unter gleichbleibenden Verhältnissen constante Verkürzung geringen Grades, welche die Ventrikelmusculatur auch während der Diastole zeigt, als Tonus der Masculatur bezeichnen, so müsste man sagen, dass die Verstärkungsfasern den Tonus erhöhen, die Hemmungsfasern ihn erniedrigen. Doch sehe ich zu einer derartigen Ausdrucksweise keinen Grund. Es stellt sich ja heraus, dass bei Reizung der Verstärkungsfasern sowohl die systolische als die diastolische Contraction des Herzmuskels wächst und bei Reizung der Hemmungsfasern sowohl die systolische als die diastolische Contraction abnimmt, beide schwinden bei längerem Herzstillstande vollständig. Beide, d. $\mathrm{h}$. die systolische wie die diastolische Zusammenziehung werden also in gleichem Sinne durch die verschiedenen Nerveneinwirkungen beeinflusst und stehen deshalb offenbar in innerem Zusammenhange.

Von Hypothesen iiber die Art der Einwirkung der Hemmungsund Verstärkungsfasern stehe ich ab. Werden doch täglich noch neue Thatsachen auf diesem Gebiete bekannt ${ }^{2}$ ), welche darthun, dass

1) Gaskel1, on the tonicity of the heart and blood vessels. Journ. of physiol. III. 1880 .

2) Vgl. Rossbach, die Erschlaffung des Herzmuskels durch nervöse und directe Reizung. Pflügers Archiv XXVII. 197. 1882. 
die Zeit zu einer Theorie der Herzinnervation noch nicht gekommen ist. Bevor das Wagniss einer solchen unternommen werden darf, sind noch Vorstudien mannigfacher Art erforderlich, die Zeit und Kräfte noch lange in Anspruch nehmen dürften.

Nur auf einen Punkt, den eigentlichen Ausgangspunkt der vorliegenden Untersuchung, sei es gestattet, mit wenigen Worten zurückzukommen. Sie kntipfte an die Frage an, ob hemmende Nervenfasern unter Umständen auch erregend wirken könnten. Für das Herz hat es sich gezeigt, dass unter den in der Vagusbahn an dasselbe herantretenden Fasern hemmende und erregende auseinandergehalten werden müssen. Es würde nun aber sehr voreilig und sehr unrichtig sein, von diesem besondern Beispiele einer Hemmungsvorrichtung allgemeine Schlüsse ableiten zu wollen. Es will mir vielmehr, je länger ich mich mit den Hemmungsvorgängen beschäftige, desto mehr geboten erscheinen, den Begriff der Hemmungsvorgänge und Hemmungsnerven genauer zu präcisiren, als es bisher geschehen. Denn als "Hemmung" sind bisher Vorgänge ganz verschiedener Art zusammengefasst worden.

Ich sehe ganz ab von Munk's ,antagonistischer" Hemmung, die für die vorliegende Erörterung ohne Belang ist, weil es sich bei derselben nur um die Auslösung einer zweiten Muskelaction neben einer ersten bandelt, durch welche die mechanischen Wirkungen der letzteren aufgehoben werden ${ }^{1}$ ). Ich habe vielmehr zwei andere Fälle im Auge, welche zu scheiden nothwendig ist, erstens die Schwächung oder Verhinderung einer centralen Erregung innerhalb der Centra, zweitens die Verhinderung der Aeusserung der centralen Erregung, ohne dass sie selbst aufgehoben wird.

In die erste Categorie gehört eine grosse Zahl der als willkürliche Hemmung und Reflexhemmung bekannten Thatsachen; wenn schon ein Theil der hierher gerechneten Fälle vielleicht unter die „antagonistische" Pseudohemmung Munk's fällt, bleibt doch eine stattliche Zahl sicherer Beispiele übrig, bei welchen es sich um Schwächung oder Aufhebung centraler Erregungsvorgänge dureh willkürliche oder peripherische sensible Einwirkung handelt ${ }^{2}$ ).

1) H. Munk, Archiv f. Anatomie und Physiologie, physiologische Abtheilung. 1881. S. 553 .

2) Vgl. R. Heidenhain, über Erregung und Hemmung. Pflügers Arch. XXVI. 596, 1881. - B. Luchsinger, ebendas. XXVII. 190. 1882. - 
Untersuchungen über den Einfluss des nv. vagus auf die Herzthätigkeit. 41

In die zweite Categorie gehört die Hemmung der Verstärkungsfasern des Herzens durch den Vagus. Denn wie früherhin Baxt bei Reizung des Vagus und accelerans der Säugethiere, jetzt ich bei electrischer und chemischer Reizung des Froschvagus gezeigt, wird durch die Hemmungsfasern des Vagus die Entwicklung der inneren Reize, welche die Erregung der Verstärkungsfasern hervorrufen, nicht aufgehoben, sondern nur ihre Aeusserung für die Wirkungsdauer der Hemmungsfasern unterdrückt. Die Vorgänge in dem motorischen Mechanismus des Herzens also, welche zur Verstärkung und Beschleunigung der Herzpulse fübren, werden nicht unmöglich gemacht, sondern nur ihre Einwirkung zeitweise suspendirt. In Fällen dieser zweiten Art müssen offenbar die Verhältnisse ganz anders liegen, als in Fällen der ersten Art; die Uebereinstimmung ist nur eine änssere, sofern als der motorische Effect nicht zu Stande kommt, aber keine innere, sofern als die Ursachen des Nichtzustandekommens verschieden sind.

Aehnlicher, aber doch in gewisser Beziehung verschiedener Art, wie bei dem Herzen, ist das Hemmungsverhältniss zwischen den gefässverengenden und den gefässerweiternden Nerven. F rey ${ }^{1}$ ) hat an den Speicheldrüsen nachgewiesen, dass bei gleichzeitiger Reizung des Sympathicus und der chorda ersterer das Uebergewicht hat, denn der Blutstrom in der Drüsenvene versiegt, weil die Ringmuskeln der Arterien sich zusammenschnüren - während bei dem Herzen bei gleichzeitger Reizung des Vagus und der Accelerantes die Musculatur erschlafft. Wie aber hier die verstärkte Thätigkeit des Herzmuskels als Nachwirkung auftritt, so dort die Beschleunigung des Blutstromes. In beiden Fällen hat der Nerv, dessen Thätigkeitsäusserung für die Dauer der Wirkung seines Antagonisten unterdrückt wurde, doch die Ansammlung von Reizursachen veranlasst, welche nach Unterbrechung der antagonistischen Wirkung zur Geltung gelangen: beim Herzen in der Pulsbeschleunigung, bei der Speicheldrüse in der Gefässerweiterung, da beide eine lange nachträgliche Geltung haben.

Nehme ich zu dem Gesagten noch hinzu, dass wir für manche Fälle über den Zusammenhang der sogenannten Hemmung noch

C. Wegele, über die centrale Natur reflectorischer Athmungshemmung. Verh. der phys.-med. Gesellsch. zu Würzburg 1882. No. 1.

1) v on Frey, über die Wirkungsweise der erschlaffenden Gefässnerven. Arbeiten der physiol. Anstalt zu Leipzig. XI. Jahrg. 1876. S. 89. 
ganz unaufgeklärt sind - ob z. B. der nv. depressor die Erregung der gefässverengenden Nerven aufhebt oder reflectorische Reizung der Gefässerweiterer veranlasst, ist meines Wissens durchans unentschieden, - so ist wohl klar, dass mit der Bezeichnung "Hemmungswirkung" nur der Hinweis auf ein äusseres Verhalten gegeben ist, dessen nähere Bestimmung und innere Charakteristik in jedem besonderen Falle näher untersucht werden muss.

Deshalb wäre es auch durchaus falsch, aus dem Nachweise, dass in dem nv. vagus die Hemmungs- und Erregungsfunction an verschiedene Classen von Nervenfasern gebunden ist, den Schluss zu ziehen, dass dieses Verhalten ein allgemeines sei. Niemand wird sich wohl entschliessen, in den Stämmen der Hautnerven reflexerzeugende und reflexverhindernde Fasern nebeneinander anzunehmen, obwohl von der Haut aus Bewegungen reflektorisch sowohl hervorgerufen als unterdrückt werden können. Die fernere Untersuchung der sogenannten Hemmungsvorgänge nach den bezeichneten Richtungen hin bildet eine der dankbarsten und interessantesten Aufgaben für die weitere Forschung.

\section{Zur Frage nach der Rindenlocalisation beim Menschen.}

Von

Prof. Sigm. Exner,

Assistenten am physiologischen Institute in Wien.

In der am 5. Jänner 1882 erschienenen Nummer der "Nature“ findet sich eine Besprechung meines jüngst erschienenen Buches „Untersuchungen über die Localisation der Functionen in der Grosshirnrinde des Menschen", Wien 1881. Dieselbe ruihrt von Ferrier her, und beurtheilt dieses Buch im Gegensatz zu mancher anderen Kritik nicht günstig. Es beruht dieses abfällige Urtheil zum grossen Theil auf Bedenken, von denen ich mir erwartet hatte, dass sie von manchen, die das Buch nicht genauer durchsähen, getheilt 


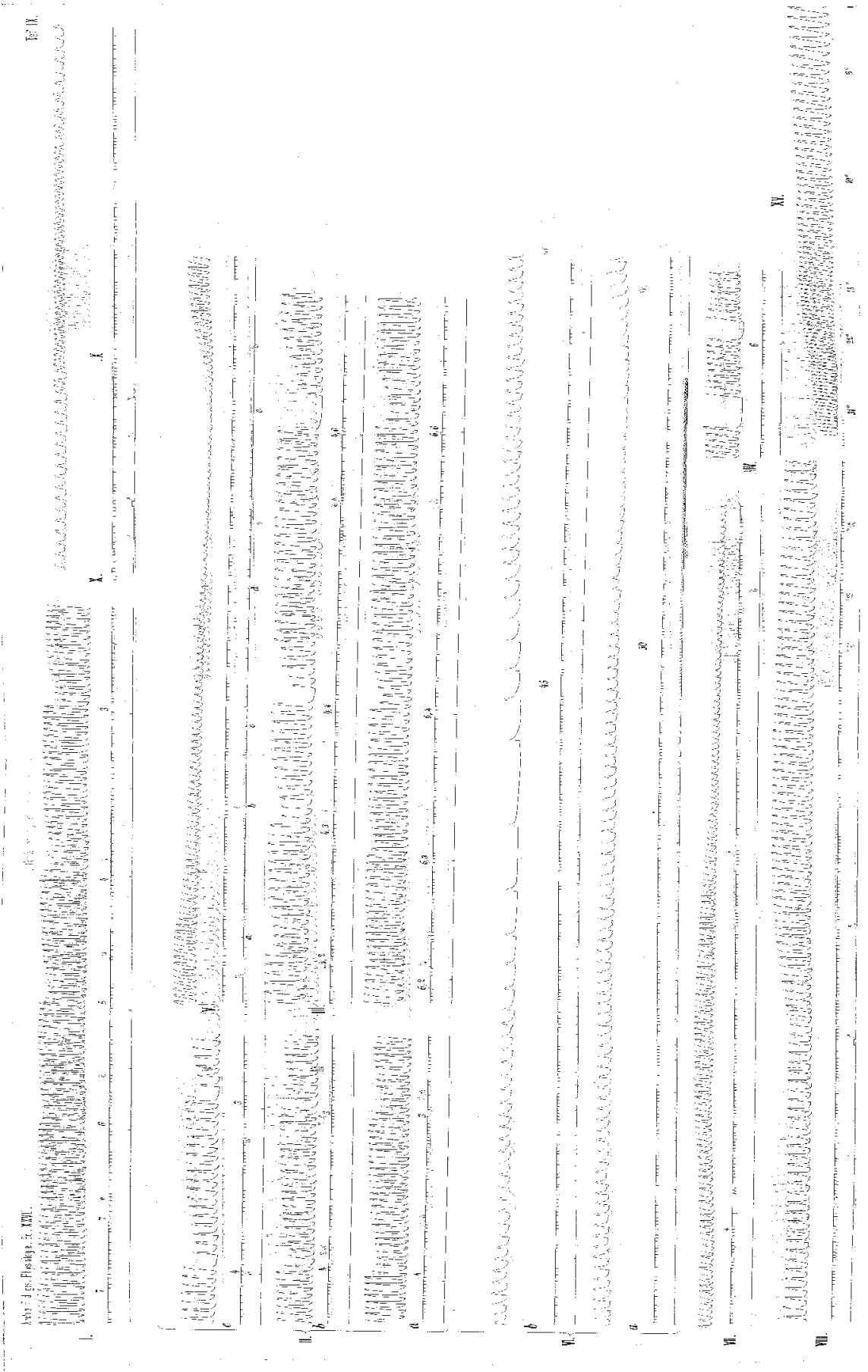




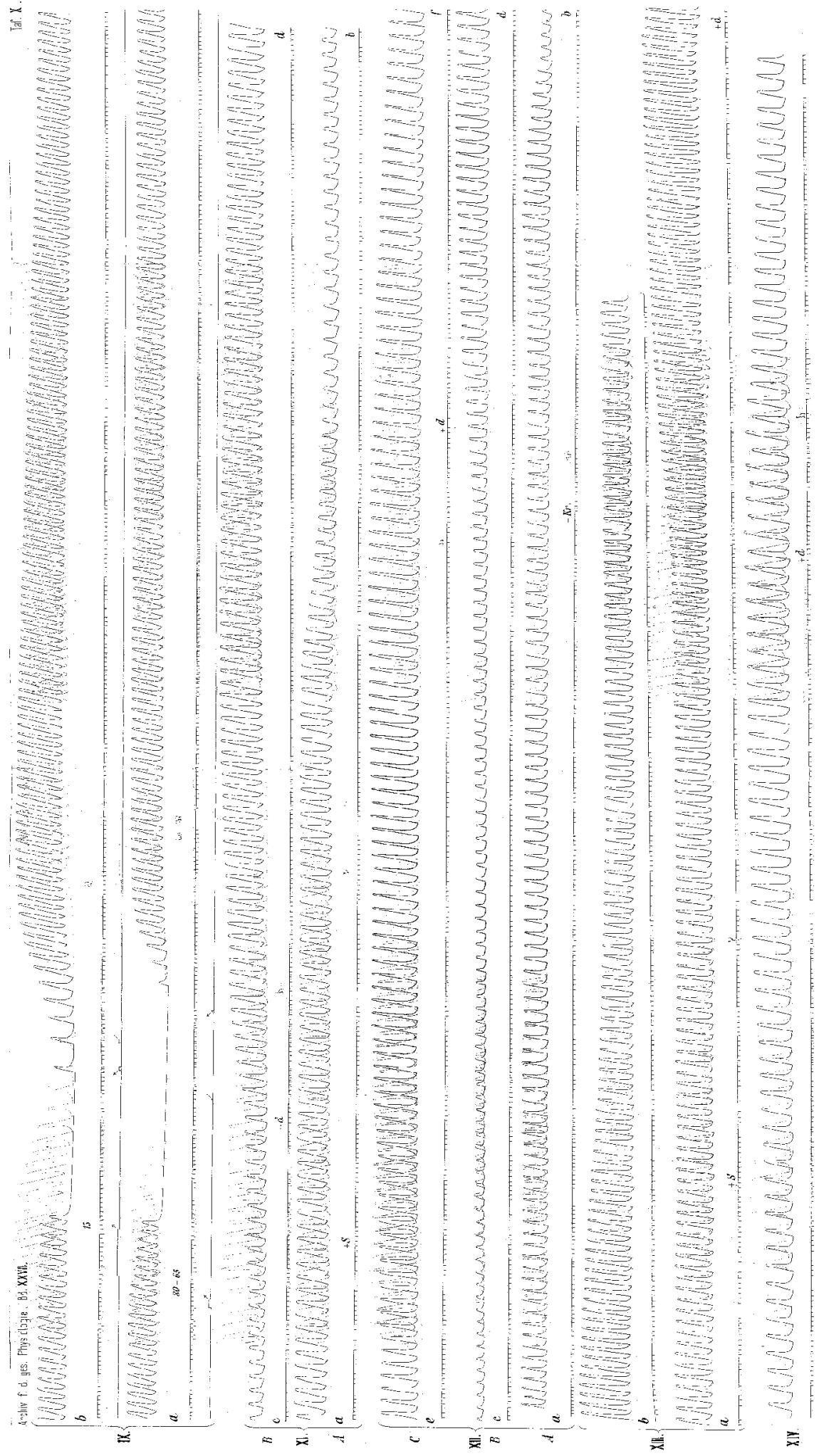

